\title{
Modulatory Effects of Metabotropic Glutamate Receptors on Local Cortical Circuits
}

\author{
Roberto De Pasquale and S. Murray Sherman \\ Department of Neurobiology, University of Chicago, Chicago, Illinois 60637
}

Glutamatergic pathways in various thalamic and cortical circuits have been classified into two types: Class 1 and Class 2, where it has been suggested that Class 1 carries the main information for processing, and Class 2 is mainly modulatory. We now extend this to the local circuitry of visual cortex of the mouse by demonstrating the modulatory actions on the Class 1 pathway from layer 4 to layers $2 / 3$ of a Class 2 input from adjacent locations in layers $2 / 3$. We found that this Class 2 input produces a long-lasting hyperpolarization and suppresses the initial responses of input from layer 4 and that this involves the postsynaptic activation of Group II metabotropic glutamate receptors. This modulation also shifts the paired pulse ratio of the layer 4 input from depression to facilitation.

\section{Introduction}

Glutamatergic pathways in thalamus and cortex have been identified as either Class 1 or Class 2, where, among other properties, Class 1 inputs exhibit paired-pulse depression and activate only ionotropic glutamate receptors, whereas Class 2 inputs exhibit paired-pulse facilitation and activate both ionotropic and metabotropic glutamate receptors (Sherman and Guillery, 2006; Sherman and Guillery, 2011). Originally, these inputs were called driver (Class 1) and modulator (Class 2), with the hypothesis that the driver inputs serve as the main information route (Sherman and Guillery, 1998). We wish to extend this classification to additional cortical circuitry starting with an important local circuit in visual cortex, the connection from layer 4 to layers $2 / 3$, which is the first intracortical step in processing the geniculocortical input (Gilbert, 1983; Callaway, 1998), and which we found to be Class 1. Since local intralaminar circuits seem likely to be modulatory (e.g., Class 2 ), we looked for and found cells in layers $2 / 3$ that received a Class 1 input from subjacent layer 4 and a Class 2 input from adjacent sites in layers $2 / 3$. Finally, we explored some modulatory actions this Class 2 input has on the Class 1 input, concentrating on the role played by Class 2 activation of metabotropic glutamate receptors. We found that activation of these receptors, which were mostly Group II, produces two modulatory actions that suppress the input from layer 4 onto cells in layers 2/3: one is a long-lasting hyperpolarization, and the other is a robust reduction of evoked EPSCs.

\footnotetext{
Received Jan. 6, 2012; revised March 30, 2012; accepted April 5, 2012.

Author contributions: R.D.P. and S.M.S. designed research; R.D.P. performed research; R.D.P. and S.M.S. analyzed data; R.D.P. and S.M.S. wrote the paper.

This work was supported by National Institute on Deafness and Other Communication Disorders Grant DC008794 to S.M.S.

The authors declare no competing financial interests.

Correspondence should be addressed to S. M. Sherman, Department of Neurobiology, University of Chicago, Abbott J-117, 947 East 58th Street, Chicago, IL 60637. E-mail: msherman@bsd.uchicago.edu.

DOI:10.1523/JNEUROSCI.0090-12.2012

Copyright $\odot 2012$ the authors $\quad 0270-6474 / 12 / 327364-09 \$ 15.00 / 0$
}

\section{Materials and Methods}

We adopted our previously described methods for slice work, laser uncaging of glutamate, and identification of cortical areas and layers (Reichova and Sherman, 2004; Lam and Sherman, 2005; Lee and Sherman, 2008; Petrof and Sherman, 2009; Lee and Sherman, 2010; Theyel et al., 2010; Covic and Sherman, 2011; De Pasquale and Sherman, 2011; Viaene et al., 2011a,b,c), and we briefly summarize them here.

Preparation and maintenance of brain slices. All animal procedures were approved by the Institutional Animal Care and Use Committee of the University of Chicago (Chicago, IL). All experiments were performed on tissue slices taken from BALB/c mice of either sex (60-65 d postnatal; Harlan). We obtained slices for study as follows. Animals were deeply anesthetized by inhalation of isoflurane (Aerrane, Baxter Pharmaceuticals) and killed. The brain was quickly removed and cooled $\left(0^{\circ} \mathrm{C}\right)$ in artificial modified CSF containing (in mM): 206 sucrose, $25 \mathrm{NaHCO}_{3}, 2.5$ $\mathrm{KCl}, 10 \mathrm{MgSO}_{4}, 1.25 \mathrm{NaH}_{2} \mathrm{PO}_{4}, 0.5 \mathrm{CaCl}_{2}$, and $11 \mathrm{D}$-glucose and oxygenated with carbogen $\left(5 \% \mathrm{CO}_{2}, 95 \% \mathrm{O}_{2}\right)$. Coronal slices were cut at a thickness of $\sim 500 \mu \mathrm{m}$ using a vibrating tissue slicer. Slices were rapidly transferred to a holding chamber with ACSF containing (in mM): 125 $\mathrm{NaCl}, 25 \mathrm{NaHCO}_{3}, 3 \mathrm{KCl}, 1.25 \mathrm{NaH}_{2} \mathrm{PO}_{4}, 1 \mathrm{MgCl}_{2}, 2 \mathrm{CaCl}_{2}$, and 25 D-glucose; the slice was then kept oxygenated for $1 \mathrm{~h}$ at room temperature $\left(\sim 25^{\circ} \mathrm{C}\right)$ before recording. In some experiments designed to block synaptic transmission, we used an ACSF containing a low $\mathrm{Ca}^{2+}$ and high $\mathrm{Mg}^{2+}$ concentration; this contained the following (in mM): $125 \mathrm{NaCl}, 25$ $\mathrm{NaHCO}_{3}, 3 \mathrm{KCl}, 1.25 \mathrm{NaH}_{2} \mathrm{PO}_{4}, 6 \mathrm{MgCl}_{2}, 0.2 \mathrm{CaCl}_{2}$, and 25 D-glucose. Slices were transferred to a recording chamber and submerged in with oxygenated ACSF that was continually perfused.

Electrophysiological recordings. Whole-cell recordings were obtained from layers $2 / 3$ neurons of $\mathrm{V} 1$ and A1. Recording pipettes were fabricated from borosilicate glass (Garner Glass) with input resistances of $\sim 6-10$ $\mathrm{M} \Omega$, and most were filled with intracellular solution containing the following (in mM): $135 \mathrm{~K}$-gluconate, $7 \mathrm{NaCl}, 10$ HEPES, $2 \mathrm{Na}_{2} \mathrm{ATP}, 0.3$ $\mathrm{Na}_{3} \mathrm{GTP}, 2 \mathrm{MgCl}_{2}, 1 \mathrm{~mm}$ DNDS (4,4'-dinitrostilbene-2,2' -disulfonate), and $0.1-0.5 \%$ biocytin, $\mathrm{pH} 7.3,290 \mathrm{mOsm}$. The DNDS blocked GABAergic inputs to the recorded cell without affecting GABAergic circuitry more generally (Dudek and Friedlander, 1996; Covic and Sherman, 2011). Thus, our recordings isolated excitatory inputs to these cells. In experiments aimed at interfering with postsynaptic action of metabotropic glutamate receptors, we added to the recording electrode the intracellular solution GDP $\beta$ S (1 mM), a nonhydrolyzable GTP ana$\log$, to block postsynaptic $\mathrm{G}$ protein-coupled activity. 
All experiments were performed on a visualized slice setup under a differential interference contrast-equipped Axioscop 2FS microscope (Carl Zeiss Instruments) Whole cell recordings were made by using a MultiClamp 700B amplifier and pClamp software (Molecular Devices). Only cells with a stable access resistance of $<20 \mathrm{M} \Omega$ were recorded. Hyperpolarizing currents were injected to identify $\mathrm{I}_{\mathrm{H}}$, while depolarizing currents were injected to identify regular, tonic or bursting spike patterns. We recorded postsynaptic responses in both current and voltage clamps.

Laser uncaging of glutamate. We used our previously described methods for uncaging of glutamate via laser illumination, a process we refer to as "photostimulation," to identify inputs connected to each recorded cell (Lam and Sherman, 2005; Covic and Sherman, 2011; De Pasquale and Sherman, 2011). Data acquisition and photostimulation were controlled by a program written in Matlab (MathWorks). Nitroindolinyl-caged glutamate (Sigma-RBI; (Canepari et al., 2001) was added to recirculating ACSF with a concentration of $0.37 \mathrm{~mm}$ during recording. Focal photolysis of the caged glutamate was accomplished by a pulsed UV laser ( 355 $\mathrm{nm}$ wavelength, frequency-tripled $\mathrm{Nd}: \mathrm{YVO} 4,100 \mathrm{kHz}$ pulse repetition rate; DPSS Laser). The intensity of the stimulus was controlled by neutral density filters. The laser beam ( $5 \mathrm{~mW}$ intensity) was directed into the side port of an Axioscop 2FS microscope using a pair of mirror galvanometers (Cambridge Technology) and then focused onto the brain slice using a low-magnification objective lens $[0 \times / 0.3$ numerical aperture $(\mathrm{NA}) /$ water UMPlanFL or $4 \times / 0.13 \mathrm{NA} /$ air UPlanFL; Olympus]. Angles of the galvanometers were computer controlled and determined the position stimulated by the laser. The optics were designed to generate a nearly cylindrical beam in the slice so as to keep the mapping two dimensional. The timing of the laser pulse for stimulation was controlled by the Q-switch of the laser and a shutter (LS3-ZM2, Vincent Associates). A variable neutral density wheel (Edmund Optics) controlled the power of the laser at different levels during experiments by attenuating the intensity of the laser. A thin microscope coverslip in the laser path reflected a small portion of the laser onto a photodiode. The current output from this photodiode was amplified, acquired by the computer and used to monitor the laser intensity during the experiment. The photodiode output was calibrated to the laser power at the back focal plane of the objective. The laser power was measured using a power meter (Thorlabs). The beam expansion was limited to a twofold gain through the scan lens/tube lens pair. The beam under-filled the objective and could be focused on a spot in the specimen plane that was around $10 \mu \mathrm{m}$ in the $x-y$ dimension and larger $(\gg 100 \mu \mathrm{m})$ in the $z$-axis. Voltage or current traces were recorded and quantified during the $100 \mathrm{~ms}$ period following UV stimulus onset using custom software.

We followed two different protocols of photostimulation. The first protocol was used to map excitatory inputs to cells of layers $2 / 3$. We assumed monosynaptic connectivity when the evoked responses had a latency $<3.5 \mathrm{~ms}$, a latency jitter of $<1 \mathrm{~ms}$, and reliably appeared with no or very rare failures. All layers and regions of nearby cortex were mapped using a preset stimulation grid. Stimulation was arranged in a sequence that maximized the distance and time between consecutive trials to avoid receptor desensitization, local caged glutamate depletion, and excitotoxicity. The second protocol was used to eliminate synaptic inputs to the recorded cell and thereby isolate direct stimulation by using the low $\mathrm{Ca}^{2+}$, high $\mathrm{Mg}^{2+}$ ACSF. Here we used a preset grid that limited stimulation to an area within $50 \mu \mathrm{m}$ from the recorded cell.

Electrical stimulation. In some experiments, we electrically stimulated inputs to the recorded layers $2 / 3$ cells by placing a bipolar concentric electrode ( $125 \mu \mathrm{m}$ pole distance; FHC) in a location guided by the uncaging maps, either in layer 4 directly below the recorded cell or in layers $2 / 3$ lateral to the cell. In all such experiments, the stimulating electrodes were placed $>100 \mu \mathrm{m}$ from the recorded cell. Electrical stimulation consisted of $0.1-0.2 \mathrm{~ms}$ pulses and ranged from 5 to $130 \mathrm{~Hz}$. We only included in our data responses recorded from inputs that we evaluated to be monosynaptic, the criteria for which included EPSP or EPSC latencies of $<3.5 \mathrm{~ms}$, a latency jitter of $<1 \mathrm{~ms}$, and no or very rare failures for stimulation levels above threshold. The intensity of stimulation was increased from subthreshold by steps of $5 \mu \mathrm{A}$ until an evident response was evoked, then intensity was further increased by $5 \mu \mathrm{A}$ for the data described here.

Pharmacology. In some experiments, we bath applied various agonists and antagonists to metabotropic glutamate receptors. The choices and concentrations used were based on our previous experience with these agents (Reichova and Sherman, 2004; Lee and Sherman, 2008; Covic and Sherman, 2011). The agents were prepared in distilled water or DMSO and diluted in ACSF to their final concentration just before use; they were delivered to the recording chamber by injecting a short bolus into the chamber flow line that was fed by a motorized peristaltic pump in a recirculating mode. Based on the chamber volume, the rate of injection, and the chamber perfusion, the ultimate bath concentration was generally estimated to be one-fourth of the initial concentration. All stimulation protocols were performed only after a minimum of $5 \mathrm{~min}$ from the bolus injection.

Agonists and antagonists to metabotropic glutamate receptors (Tocris Bioscience) were applied at the following concentrations: the general metabotropic glutamate receptor agonist ACPD (1-amino-1,3cyclopentanedicarboxylic acid) at $100 \mu \mathrm{M}$; the Group I metabotropic glutamate receptor agonist DHPG $[(R S)$-3,5-dihydrophenylglycine] at $100 \mu \mathrm{m}$; the Group II metabotropic glutamate receptor agonist APDC [(2R,4R)-4-aminopyrrolidine-2,4-dicarboxylate] at $100 \mu \mathrm{M}$; the type 1 metabotropic glutamate receptor antagonist LY367385 [(S)-(+)- $\alpha-$ amino-a-methylbenzeneacetic acid] at $50 \mu \mathrm{m}$; type 5 metabotropic glutamate receptor antagonist MPEP (2-methyl-6-(phenylethynyl)pyridine) at $30 \mu \mathrm{M}$ (LY367385 and MPEP were added together to the bath to block Group I metabotropic glutamate receptors, which consisted of types 1 and 5); and the Group II metabotropic glutamate receptor antagonist LY341495 [(2S)-2-amino-2-[(1S,2S)-2-carboxycycloprop-1-yl]-3(xanth-9-yl) propanoic acid] at $100 \mu \mathrm{M}$. Antagonists to ionotropic glutamate receptors (Tocris Bioscience) were also used: the AMPA receptor antagonist DNQX (6,7-dinitroquinoxaline-2,3-dione) at $50 \mu \mathrm{M}$; and the NMDA receptor antagonist MK-801 [(+)-5-methyl-10,11-dihydro$5 \mathrm{H}$-dibenzo[a,d]cyclohepten-5,10-imine maleate] at $40 \mu \mathrm{M}$.

Normalization and statistical procedures. In many of our analyses, EPSC values $\left(\right.$ EPSC $_{\mathrm{x}}$ ) have been normalized to the mean value measured in the control condition ( EPSC $_{\text {control }}$ ) according to the following procedure: $\mathrm{EPSC}_{\text {normalized }}=\left(\mathrm{EPSC}_{\mathrm{x}} / \mathrm{EPSC}_{\text {control }}\right)$, expressed as a percentage. The effect of each agonist was evaluated using the one-way repeated measures (RM)-ANOVA on the three experimental conditions of control, agonist effect, and wash out. The effect of modulation via highfrequency stimulation was evaluated using a paired $t$ test comparing the control condition to the modulation condition.

\section{Results}

In our experiments we did not distinguish between layers 2 and 3 and simply refer to these as "layers $2 / 3$." We performed our recordings from a total of 154 cells: 127 from V1, and 24 from A1. Neurons of V1 had resting membrane potentials of $-66.4 \pm 2.3$ $\mathrm{mV}$ and input resistances of $209.1 \pm 56.3 \mathrm{M} \Omega$. The respective values for neurons of A1 were $-64.1 \pm 6.2 \mathrm{mV}, 235.2 \pm 75.1$ $\mathrm{M} \Omega$. Two of the neurons were fast spiking, and the rest had a regular spiking pattern. In the data described, those for the two fast spiking neurons were not statistically different from those of the other cells. Thirteen of the recorded neurons, all regular spiking, were successfully filled with biocytin and proved to be pyramidal cells. We found no evidence in our sample of layers $2 / 3$ cells that clearly divided them into different types, since all of the parameters we measured fell along a continuum.

\section{Recordings from V1}

\section{Synaptic properties of intracortical inputs to layers $2 / 3$}

We determined the main local sources of input to recorded cells in layers $2 / 3$ via laser photostimulation to uncage glutamate (Callaway and Katz, 1993; Lam and Sherman, 2005). We refer to this approach below simply as photostimulation. We then applied bipolar stimulating electrodes to the region from which such 

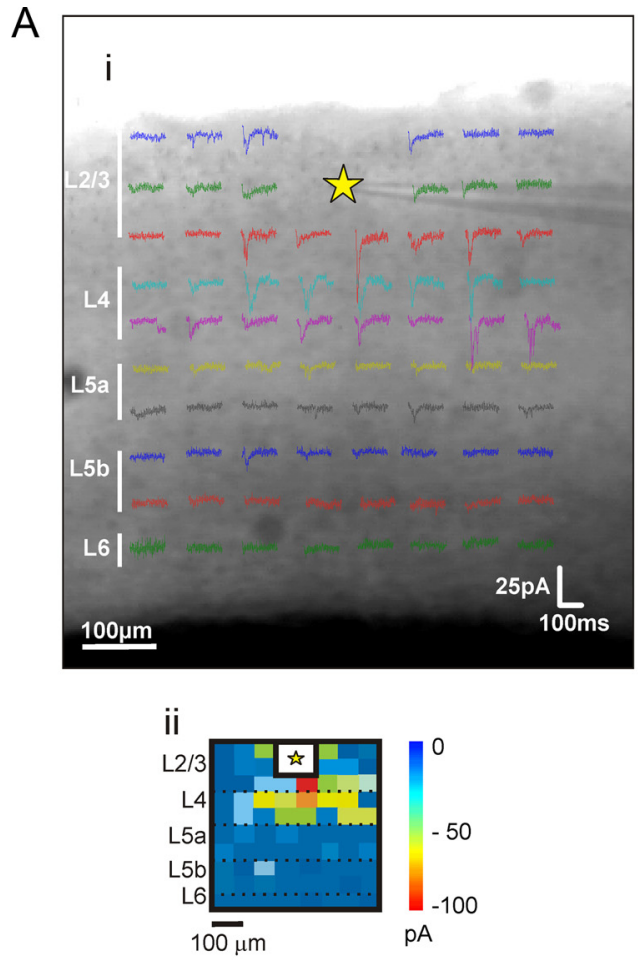

$\mathrm{E}$

Layer 4 stimulation
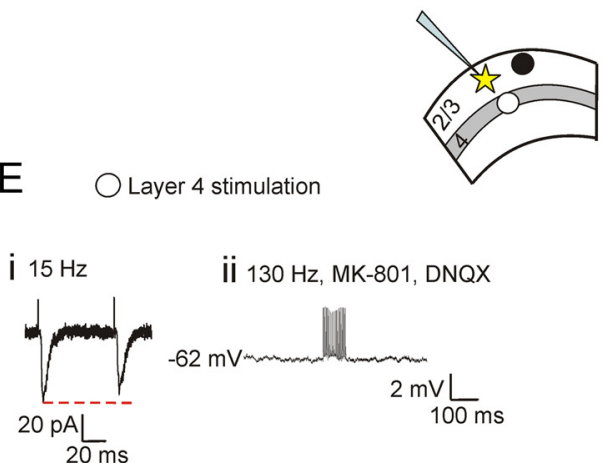

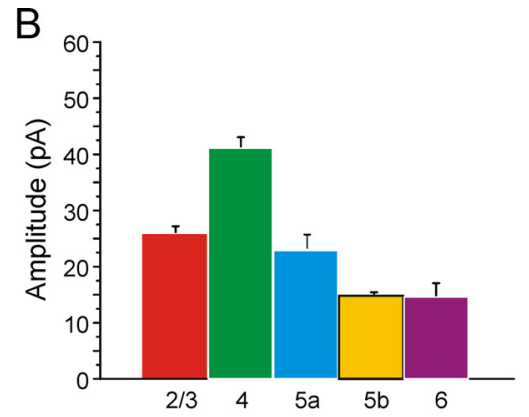

C

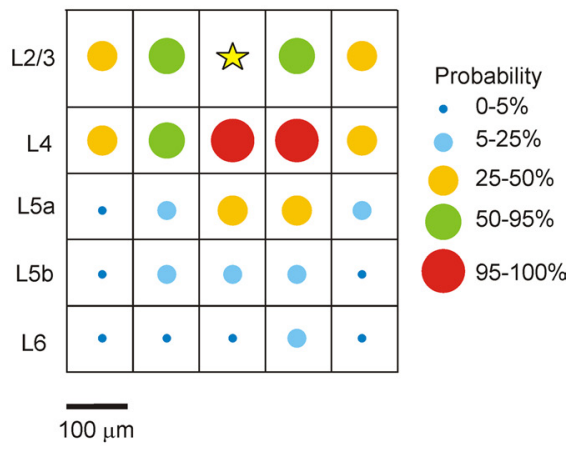

$\mathrm{F}$

Layer 2/3 stimulation

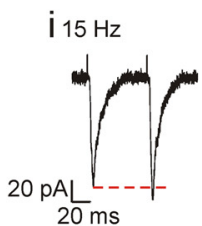

ii $130 \mathrm{~Hz}, \mathrm{MK}-801, \mathrm{DNQX}$

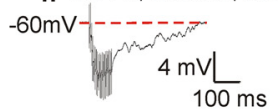

iii $130 \mathrm{~Hz}, \mathrm{MK}-801, \mathrm{DNQX}$

LY 341495

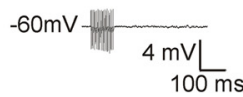

Figure 1. Synaptic properties of principal intracortical projections to layers $2 / 3$ in V1.A-C, Profile of connectivity. The principal intracortical projections to neurons in layers $2 / 3$ come from layers $2 / 3$ and $4 . A$, Example of a photostimulation map in V1 (Ai) also shown in false color (Aii). The recording site in V1 is indicated by the yellow star. $\boldsymbol{B}$, Average amplitude of synaptic responses evoked in different laminar regions of V1. C, Matrix showing the probability of getting significant responses in different regions of V1; recorded cell is indicated by yellow star. D, E, Synaptic properties of inputs coming from layers 2/3 and 4. D, Placement of concentric bipolar electrodes in layer 4 (white circle) and layers $2 / 3$ (black circle). E, Stimulation in layer 4 elicits paired-pulse depression (Ei). High-frequency stimulation fails to evoke metabotropic activation during bath application of ionotropic glutamate receptor antagonists (Eii). $\boldsymbol{F}$, Stimulation in layers $2 / 3$ elicits paired-pulse facilitation (Fi). High-frequency stimulation (130 Hz) in the presence of ionotropic glutamate receptor antagonists evokes a hyperpolarizing response (Fii) that is blocked by a Group II metabotropic glutamate receptor antagonist (Fiii).

inputs were mapped to further study synaptic inputs to the recorded cells.

Photostimulation. Local inputs were mapped by photostimulation for 10 cells in layers $2 / 3$. Within roughly $50 \mu \mathrm{m}$ of the recorded neuron, the evoked inward currents had a shorter latency and larger amplitude, often exceeding $100 \mathrm{pA}$, and we interpreted this as direct stimulation of the recorded cell. This interpretation is supported by experiments described below in which direct responses to photostimulation were isolated by applying a bath of low $\mathrm{Ca}^{2+}$ and high $\mathrm{Mg}^{2+}$ concentrations. We thus omitted this proximal area from analysis of inputs (Fig. 1Ai,ii). As a result, our measurements of layers $2 / 3$ connections only included inputs at least $50 \mu \mathrm{m}$ away from each recorded neuron. Every recorded cell in layers $2 / 3$ showed the same pattern of local inputs as illustrated in Figure $1 \mathrm{~A} i$ : these inputs arrived principally from immediately subjacent layer 4 and also via horizontal connections from other nearby layers $2 / 3$ cells; a minor input arrived from layer $5 \mathrm{a}$ (Fig. $1 A-C, \mathrm{~L} 5 \mathrm{a}$ ). This profile of connectivity is consistent with what has previously been reported for pyramidal neurons in rat visual cortex (Dantzker and Callaway, 2000).

Electrical stimulation. Because of the consistency of the input patterns revealed by photostimulation, we felt that we could reliably activate the local inputs to the recorded cells in layers $2 / 3$ by placing stimulating electrodes in the appropriate regions without further photostimulation; to activate layer 4 inputs, we placed the electrodes just below the recorded cell in layer 4, and to activate the horizontal inputs, we placed the electrodes roughly $100 \mu \mathrm{m}$ to one side of the recorded cell. In every case, this led to evoked EPSCs (or EPSPs) with properties consistent with monosynaptic activation as defined above in Materials and Methods. The synaptic properties we tested are those we have used previously and have proven adequate to identify glutamatergic inputs as Class 1 
A
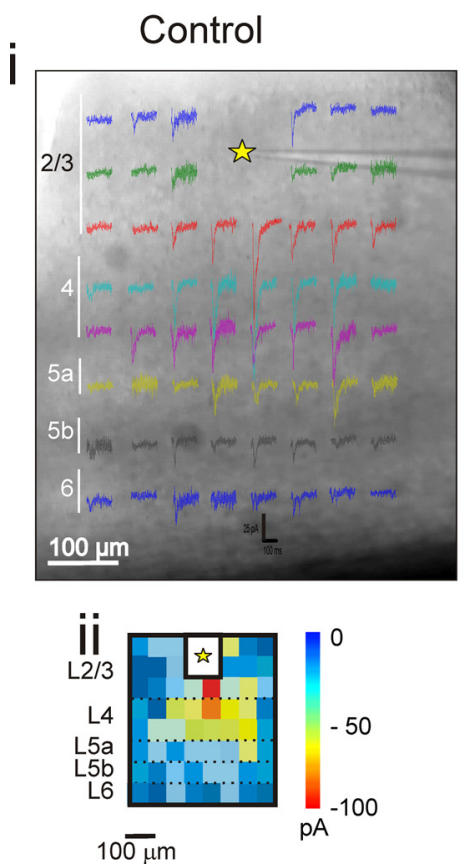

$10 \bar{\mu} \mathrm{m}$
$\mathrm{B}$
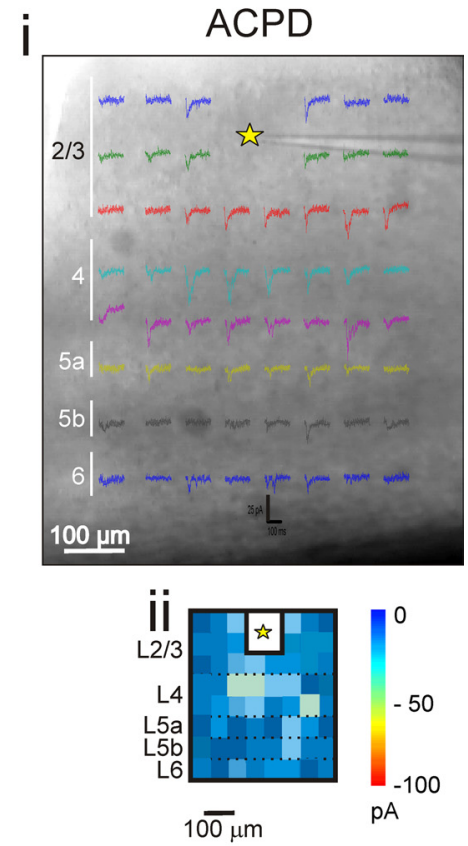

C

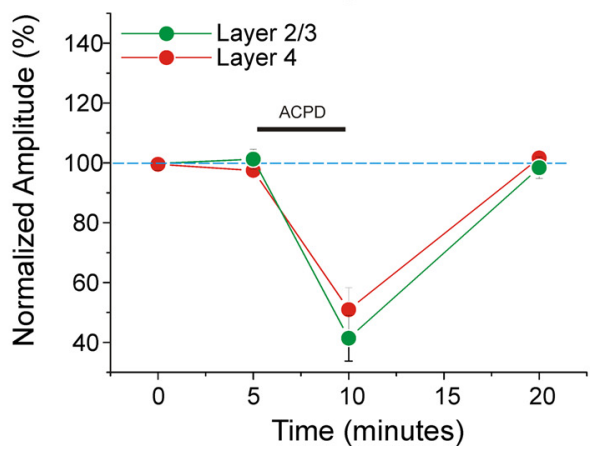

Figure 2. $\quad A C P D$ decreases synaptic responses evoked in layers $2 / 3$ of $\mathrm{V} 1$ by photostimulation. $A, B$, Example of a photostimulation map in $\mathrm{V} 1$ before $(\boldsymbol{A})$ and after $(\boldsymbol{B})$ bath application of $A C P D$. The recording site in $\mathrm{V} 1$ is indicated by the yellow star, and false color maps are shown below (Aii, Bii). C, The graph shows the effects of ACPD on inputs from layers $2 / 3$ and 4 . Normalized EPSC amplitudes of evoked responses are shown before, during, and after the application of the agonist. ACPD was administrated after a stable base line period of $5 \mathrm{~min}$. EPSC amplitudes are normalized to the mean of the base line values.

or Class 2 (Sherman and Guillery, 1998, 2006; Covic and Sherman, 2011; Viaene et al., 2011a). These properties include a measure of paired-pulse depression or facilitation and the presence or absence of a metabotropic glutamate receptor component to the response.

Figure $1 D$ shows a diagram of the location of the recorded cell in layers 2/3 (star) and stimulation locations in layers $2 / 3$ (filled circle) or layer 4 (open circle) for electrical activation experiments. The input from layer 4 was thus studied in 10 neurons recorded in layers $2 / 3$, and the results were quite consistent since this input for all cells was Class 1 . When stimulated at $15 \mathrm{~Hz}$, these inputs showed paired-pulse depression, meaning that the first evoked EPSC was followed by an EPSC of smaller amplitude (Fig. $1 \mathrm{Ei})$. High-frequency stimulation $(130 \mathrm{~Hz})$ in the presence of AMPA and NMDA blockers failed to elicit any response, indicating no metabotropic glutamatergic component response (Fig. 1 Eii). We likewise tested the responses to activation of horizontal connections within layer $2 / 3$ in 10 other neurons recorded in layers $2 / 3$, and each of these consistently showed a Class 2 profile. Stimulation at 15
$\mathrm{Hz}$ evoked paired-pulse facilitation, meaning that the first EPSC was followed by an EPSC of larger amplitude (Fig. $1 \mathrm{Fi}$ ). When AMPA and NMDA antagonists were applied, stimulation at $130 \mathrm{~Hz}$ in current clamp evoked a slow hyperpolarization (Fig. 1 Fii) that was blocked with an antagonist to Group II metabotropic glutamate receptors (Fig. 1 Fiii). Among the horizontal inputs from layers $2 / 3$, eight showed a hyperpolarizing response activated by Group II metabotropic glutamate receptors, and the other two showed a depolarizing response activated by type 1 metabotropic glutamate receptors; this was because these responses were blocked by the specific antagonist LY367385 (data not shown).

Although not relevant to this study, in other pathways we have shown subtypes to the Class 1 group (Reichova and Sherman, 2004; Lee and Sherman, 2008; Petrof and Sherman, 2009; Covic and Sherman, 2011; De Pasquale and Sherman, 2011; Viaene et al., 2011a,b,c). Class 1A inputs have a strictly all-or-none activation pattern whereas Class $1 \mathrm{~B}$ inputs have a pattern intermediate between all-or-none and the graded pattern seen in Class 2 inputs, and Class 1C inputs have a curious pairedpulse pattern whereby the first two EPSPs show facilitation and the remainder in a train show depression. The layer 4 to layers $2 / 3$ input of this study by this further subclassification is Class 1B. These subclasses are not further considered here.

Effects of activating glutamatergic metabotropic receptors on synaptic responses evoked in layers $2 / 3$

Because Class 2 inputs activate metabotropic glutamate receptors, we investigated the role of such activation on responses to synaptic inputs. We did this by testing the effects of metabotropic glutamate receptor activation on EPSCs evoked in layers $2 / 3$ cells on two inputs: one from layers $2 / 3$ and the other from layer 4 . We activated metabotropic glutamate receptors in two complementary ways: by applying agonists and by using high-frequency stimulation of other Class 2 inputs from layers $2 / 3$.

Agonist application. Figure 2 shows the effects of application of the general metabotropic glutamate receptor agonist ACPD on EPSCs evoked in cells of layers $2 / 3$ by photostimulation. The agonist was administrated for $5 \mathrm{~min}$, and EPSCs were recorded before, during, and after the drug application for six cells (Fig. $2 A, B)$. ACPD caused a roughly $50 \%$ decrease of EPSC amplitudes for both layers $2 / 3$ and 4 inputs to layers $2 / 3$ (Fig. $2 ; p<$ 0.001 on a repeated measures ANOVA test for each input). We next used the following strategy to determine whether the effects of ACPD application were presynaptic or postsynaptic. We reasoned that if the effects were postsynaptic, this would imply a reduced postsynaptic response to transmitter release, and we could mimic this by monitoring the effects of ACPD on direct 
A Low $\mathrm{Ca}^{2+} / \mathrm{High} \mathrm{Mg}^{2+} \mathrm{ACSF}$

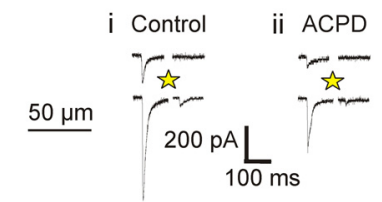

B

Low $\mathrm{Ca}^{2+} / \mathrm{High} \mathrm{Mg}^{2+} \mathrm{ACSF}+\mathrm{GDP} \beta S$
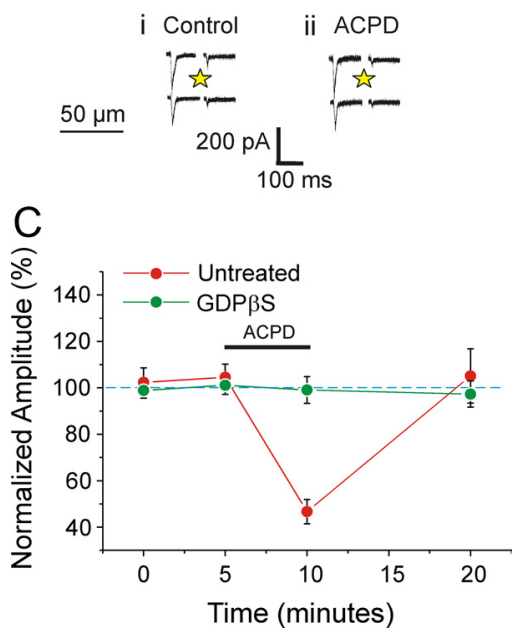

Figure 3. Effects of ACPD on direct photostimulation of neurons in layers $2 / 3$ in $\mathrm{V} 1 . A, B$, Example of experiments of direct photostimulation on the recorded neuron when synaptic transmission is blocked by a low $\mathrm{Ca}^{2+}$ and high $\mathrm{Mg}^{2+}$ bathing medium. Photostimulation evokes large inward currents (Ai) that are notably reduced after ACPD bath application (Aii). ACPD has no effect when the recorded neuron has been infused with GDP $\beta S$ ( $B \boldsymbol{B}$, ii). $\boldsymbol{C}$, Graph showing the effects of ACPD on direct responses. Amplitudes are normalized to the mean of the base line values. ACPD fails to affect cells treated with $\operatorname{GDP} \beta S(p<0.001$ on a $t$ test).

photostimulation of layers $2 / 3$ cells. To isolate postsynaptic responses, these experiments were carried out on four neurons in layers $2 / 3$ in a low $\mathrm{Ca}^{2+}$ and high $\mathrm{Mg}^{2+}$ bathing medium to block any synaptic transmission. As shown in Figure $3, A$ and $C$, under these conditions ACPD application strongly decreased direct responses by roughly $50 \%$ ( $p<0.001$ on a repeated measures ANOVA test). As a complementary test for the site of the agonist action, we observed the effects of GDP $\beta$ S administered intracellularly via the recording electrode on four other neurons in layers $2 / 3$ in the low $\mathrm{Ca}^{2+}$ and high $\mathrm{Mg}^{2+}$ bathing medium following the same protocol of ACPD administration. GDP $\beta$ S blocks G-protein activity postsynaptically and thus should interfere with postsynaptic actions of ACPD. This application of GDP $\beta$ S cancelled the effects of ACPD application, and the difference with and without GDP $\beta$ S is statistically significant (Fig. $3 B, C$; $p<$ 0.001 on a $t$ test). Together, these data strongly support a postsynaptic site of activation of the ACPD-induced reduction of EPSCs onto cells in layers 2/3.

Activation of metabotropic glutamate receptors is known to affect short term plasticity (O'Leary et al., 1997; Gerber et al., 2000; Bandrowski et al., 2002). We thus studied the action of ACPD on trains of stimuli, focusing on the input from layer 4 to layers $2 / 3$. We recorded such EPSCs from 11 neurons in layers $2 / 3$. Short-term plasticity was tested by delivering four pulses at $15 \mathrm{~Hz}$ at the layer 4 input site. ACPD caused a significant, roughly $40 \%$ decrease of the first evoked EPSC $(p<0.001$ on a repeated measures ANOVA), but the following three were unaffected (Fig. $4 A, C ; p>0.1$ on a repeated measures ANOVA). As a result, the paired-pulsed effects, as measured by comparing the amplitudes of the first two evoked EPSCs, shifted from depression to facilitation (Fig. $4 \mathrm{C} ; p<0.001$ on a repeated measures ANOVA). We repeated this experiment on eight other cells with GDP $\beta$ S applied to the recorded cell to provide further evidence for the site of action of this effect. In these cells, ACPD failed to produce a change in the amplitude of any of the four evoked EPSCs (Fig. $4 D ; p>0.1$ on a repeated measures ANOVA), again supporting a postsynaptic site for these effects of ACPD.

We investigated the possibility that metabotropic glutamate receptor application might have different effects at different stimulation frequencies. We thus studied the effects of ACPD application on EPSCs evoked in 14 cells in layers $2 / 3$ by stimulation of layer 4 inputs at four different interstimulus intervals (ISIs; 10, $40,66,100$, and $200 \mathrm{~ms}$ ). Figure $4 C$ summarizes the results. Without ACPD, the paired-pulse ratio exhibits depression at short ISIs $(p<0.001$ on a repeated measures ANOVA). Application of ACPD prevents paired-pulse depression at all ISIs (Fig. 4C).

We performed two sets of experiments to identify the metabotropic glutamate receptors involved in these effects by using more specific agonists than ACPD. The specific Group I agonist DHPG was tested on eight cells in layers $2 / 3$, and this failed to affect EPSCs evoked from layer 4 (Fig. $4 D ; p>0.1$ on a repeated measures ANOVA). Like ACPD, the specific Group II agonist APCD induced a significant decrease on the first EPSC $(p<0.01$ on a repeated measures ANOVA) but did not affect the following three EPSCs (Fig. $4 D ; p>0.1$ on a repeated measures ANOVA). We thus conclude that the main effect of reducing EPSC amplitude on layers $2 / 3$ cells is produced through postsynaptic activation of Group II metabotropic glutamate receptors.

Activation of metabotropic glutamate receptors via highfrequency stimulation. Above, we document the effects of using agonists to activate Group II metabotropic glutamate receptors on layers $2 / 3$ cells. Here, we sought to determine whether these same metabotropic glutamate receptors activated by highfrequency stimulation of a Class 2 input to layers $2 / 3$ cells have the same effects. Given the results of uncaging experiments reported above and in Figure 1, we targeted nearby locations in layers $2 / 3$, which innervate layers $2 / 3$ cells with a Class 2 input involving Group II metabotropic glutamate receptors. We thus recorded from nine neurons following the protocol shown in Figure $5 \mathrm{~A}$. Two concentric bipolar stimulating electrodes were used, one applied in layer 4 (referred to as "S4") just below the recorded cell in layers $2 / 3$, and the other nearby in layers $2 / 3$ (referred to as "S2/3"). In the Control condition, we stimulated S4 only with four pulses at $15 \mathrm{~Hz}$ (Fig. 5Aii). In the Modulation condition, S2/3 was stimulated first (20 pulses, $130 \mathrm{~Hz}$ ), followed by stimulation of S4 (4 pulses, $15 \mathrm{~Hz}$ ) $20 \mathrm{~ms}$ after the last stimulation pulse of S2/3 (Fig 5Aiii). The outward current caused by Group II metabotropic glutamate receptor activation is largely obscured by activation of ionotropic glutamate receptors in the example of Figure 5Aiii; this is clear when comparing the effects of the highfrequency stimulation before (Fig. 5Aiii, black trace) and after (red trace) an antagonist for the metabotropic glutamate receptors is applied. In the Modulation condition, the first EPSC from S4 was significantly reduced by about $50 \%$ (Fig. $5 C, D ; p<0.01$ on a paired $t$ test). No difference was found for the following three EPSCs (Fig. $5 D ; p>0.1$ on a paired $t$ test). Thus, the paired-pulse ratio was significantly increased (Fig. $5 E ; p<0.01$ on a paired $t$ test). Application of the Group II metabotropic glutamate receptor antagonist LY341495 abolished these effects of S2/3 stimulation (Fig. $5 D, E ; p>0.1$ on a $t$ test). The above protocol was repeated for 10 trials alternating between Control and Modula- 
A

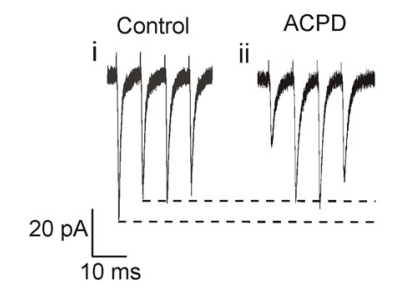

C

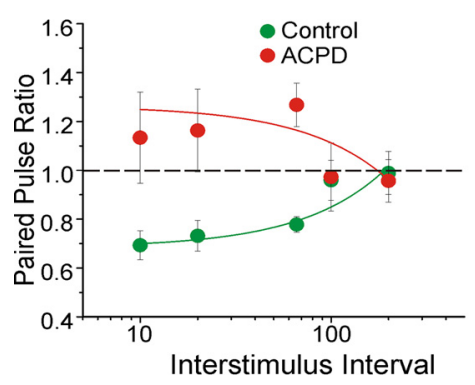

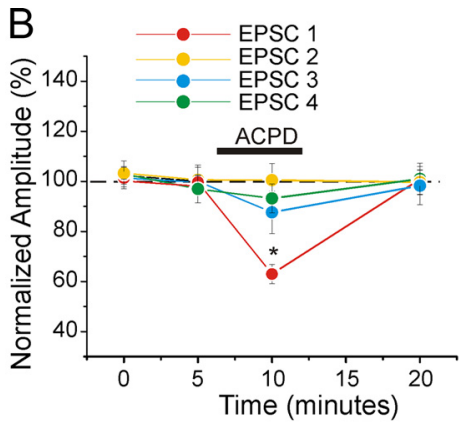

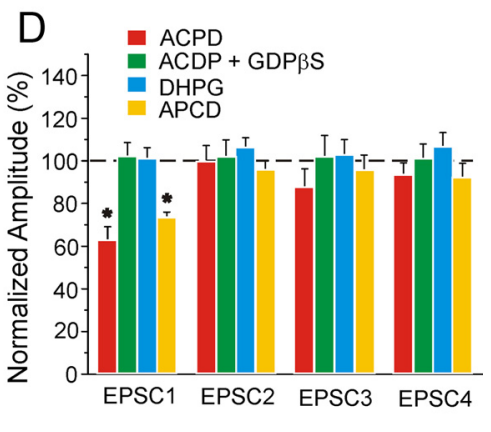

Figure 4. Effects of metabotropic glutamate receptor agonists on the synaptic properties of inputs from layer 4 to layers $2 / 3$ in V1. $A, B$, Effects of ACPD on synaptic responses evoked by four pulses delivered at $15 \mathrm{~Hz}$. $\boldsymbol{A}$, Traces showing an example experiment. $\boldsymbol{B}$, Graph showing the normalized amplitudes of the four EPSCs before, during, and after ACPD application. Only the first EPSC is significantly decreased ( $p<0.001$ on a RM-ANOVA). C, ACPD prevents paired-pulse depression. Graph shows the effects of ACPD on paired-pulse ratio (the amplitude of the second EPSC divided by that of the first) at different interstimulus intervals. Normally, paired-pulse depression increases with a decreasing interstimulus interval ( $p<0.001$ on a RM-ANOVA). With ACPD, such depression is abolished at all intervals ( $p>0.1$ on a RM-ANOVA). D, ACPD acts postsynaptically and through the activation of Group II metabotropic glutamatergic receptors. Graph showing the normalized amplitudes of the four EPSCS (15 Hz) during the administration of ACPD, DHPG, and APCD. The effect of ACPD on neuron treated with GDP $\beta$ S is also reported. $A C P D$ and $A P C D$, but not DHPG, cause a significant decrease only on the first EPSC ( $p<0.001$ on a RM-ANOVA, $p<0.01$ on a RM-ANOVA, and $p>0.1$ on a RM-ANOVA, respectively). The effect of ACPD is prevented in cells treated with GDP $\beta S$ ( $p>0.1$ on a RM-ANOVA).

tion during several $5 \mathrm{~s}$ intervals as indicated in Figure $5 C$. Prior modulation did not affect the subsequent control condition $(p>$ 0.1 on a RM-ANOVA). Finally, as above with agonist application, we tested the effects on 10 other cells in layers $2 / 3$ following the same protocol just described but with GDP $\beta S$ in the recording pipette. We found that in the Modulation condition only the first response was significantly affected (Fig. 5D; $p<0.05$ on a paired $t$ test), but the effect was much smaller (roughly 10\%) than that seen without GDP $\beta S$ (roughly 50\%), and this difference was significant $(p<0.0001$ on a $t$ test). This again indicates that the modulation evoked by stimulation of $\mathrm{S} 2 / 3$ is prevalently postsynaptic.

The evidence that these effects involve activation of postsynaptic Group II metabotropic glutamate receptors suggests that other inputs to $2 / 3$ in addition to those from layer 4 could be affected by high-frequency stimulation of layers $2 / 3$ inputs to layers $2 / 3$ cells. Accordingly, we recorded from 10 neurons using the same protocol described above with the only difference being that both stimulating electrodes were placed on layers $2 / 3$ on opposite sides of the recording electrode. To one of the stimulating electrodes we applied highfrequency stimulation, while to the other we applied test pulses as from the S4 site described above. In the Modulation condition the first three EPSCs were significantly reduced with respect to the Control condition (Fig. $6 A ; p<0.01$ on a paired $t$ test for the first two EPSCs and $p<0.05$ on a paired $t$ test for the third EPSC). Since the first two responses were similarly depressed, our measure of pairedpulse effects showed no detectable effect (Fig. $6 B ; p>0.1$ on a paired $t$ test). Bath application of LY341495 prevented the effect of high- frequency stimulation (Fig. $6 A ; p>0.1$ on a $t$ test for all EPSCs), confirming that the effects seen here are due to activation of Group II metabotropic glutamate receptors. Finally, we applied the same experimental protocol to 11 other neurons with GDP $\beta S$ in the recording pipette. In the Modulation condition, the amplitude of all responses for these cells was not statistically different from that in the Control condition (Fig. $6 A ; p>$ 0.1 on a paired $t$ test).

\section{Recordings from A1}

To determine the potential generality of the effects reported above for V1 and illustrated in Figure 3, we repeated a select subset of the experiments in A1. In four cells from layers $2 / 3$, we tested the effects of ACPD on activation by photostimulation in a low $\mathrm{Ca}^{2+}$ and high $\mathrm{Mg}^{2+}$ bath solution as described previously for $\mathrm{V} 1$ (see above, Recordings from V1, Effects of activating glutamatergic metabotropic receptors on synaptic responses evoked in layers 2/3). Direct responses were significantly decreased by ACPD $(p<0.001$ on repeated measures ANOVA), and the decrease of roughly $50 \%$ was similar to that reported for V1 (Fig. 7A). We also investigated EPSCs evoked from layer 4 in 10 other cells in layers $2 / 3$ and the effect of conjoint activation of metabotropic glutamate receptors by delivering highfrequency stimulation to nearby locations in layers $2 / 3$. We adopted the same proto$\mathrm{col}$ as described above for V1 and illustrated in Figure 5. Modulation via high-frequency stimulation significantly decreased the first two evoked EPSCs ( $p<0.01$ on a paired $t$ test). Bath application of LY341495 prevented this effect $(p>0.1$ on a $t$ test), demonstrating the involvement of Group II metabotropic glutamate receptors. Modulation was tested further in 10 additional cells in layers $2 / 3$ with GDP $\beta S$ in the electrode. In these experiments, modulation slightly decreased the first response $(p<0.05$ on a paired $t$ test) and increased paired-pulse ratio $(p<0.01$ on a paired $t$ test), revealing the possible presence of a minor presynaptic component. A direct comparison between the effects of modulation in $\mathrm{A} 1$ and $\mathrm{V} 1$ shows no significant difference between the two areas (Fig. $7 B$ ). Neither the treatment with GDP $\beta S$ nor the addition of Group II metabotropic glutamate receptor antagonists had significantly different results between A1 and V1 ( $p>0.1$ on a $t$ test for all tests).

\section{Discussion}

We have shown two distinct local glutamatergic inputs to cells in layers 2/3 of mouse cortex: one from subjacent layer 4 is Class 1 , and one from adjacent layers $2 / 3$ is Class 2 . Furthermore, when Group II metabotropic glutamate receptors are activated from the adjacent Class 2 inputs in layers $2 / 3$, synaptic inputs to the target cells in layers $2 / 3$ are suppressed by two independent mechanisms, one being a prolonged IPSP, and the other a reduction of initially evoked EPSPs, the latter also resulting from a so-far unspecified postsynaptic action of metabotropic glutamate recep- 
tors. Thus, one of the modulatory actions of the Class 2 input from adjacent sources to layers $2 / 3$ cells is a suppression of the Class 1 input from layer 4 . Given the prevailing view that an information pathway through cortex starts with thalamic input to layer 4 and passes from there to layers 2/3 (Gilbert, 1983; Callaway, 1998), this finding demonstrates one means of modulating that information route. We observed the main effects in both visual and auditory cortices, suggesting the possibility that this may be a general property of cortical circuitry. One curious feature of this effect of activation of Group II metabotropic glutamate receptors is that, at higher frequency stimulation rates, only the initial EPSC is affected. We as yet have no explanation for this phenomenon, but a similar effect has been reported previously (Bandrowski et al., 2002). This suggests that the effects on EPSCs seen here are more pronounced at lower frequencies of excitatory input.

\section{Class 1 and 2 inputs}

We have recently reviewed the properties of glutamatergic Class 1 and 2 inputs in thalamic and cortical circuitry (Sherman and Guillery, 2011). Compared to Class 2 inputs, Class 1 inputs have larger initial EPSPs, show a generally depressing pairedpulse pattern indicating a higher probability of transmitter release, are activated in a less graded pattern, and activate only ionotropic glutamate receptors, whereas Class 2 inputs also activate metabotropic glutamate receptors. These two classes have been seen in various inputs to thalamus (Reichova and Sherman, 2004; Petrof and Sherman, 2009; Lee and Sherman, 2010; Theyel et al., 2010), in different thalamocortical pathways (Lee and Sherman, 2008; Theyel et al., 2010; Viaene et al., 2011a,b,c), in connections between cortical areas (Covic and Sherman, 2011; De Pasquale and Sherman, 2011), and in intrinsic cortical pathways (Lee and Sherman, 2009). No other type of glutamatergic input has so far been seen in any of these thalamic or cortical pathways. Furthermore, as indicated by Sherman and Guillery (2011) in their Figure 2, the specific parameters of each of the Class 1 or 2 cortical and thalamic circuits are consistent across thalamic and cortical circuitry. Our current data merely add to this scheme with further examples: a Class 1 input from layer 4 to layers $2 / 3$, and a lateral pathway within layers $2 / 3$ that is Class 2 .

It is remarkable that, so far, we have found that all glutamatergic inputs in thalamus and cortex can be identified as either Class 1 or Class 2 (Reichova and Sherman, 2004; Lee and Sherman, 2008, 2009, 2010; Covic and Sherman, 2011; De Pasquale and Sherman, 2011; Viaene et al., 2011a,b,c). Obviously, as more

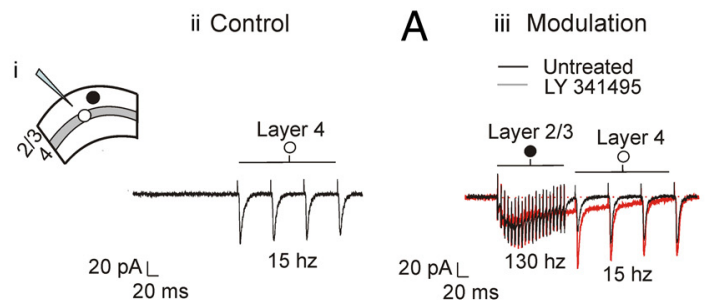

B

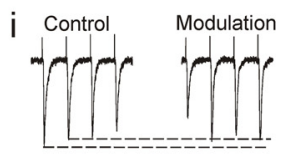

Untreated

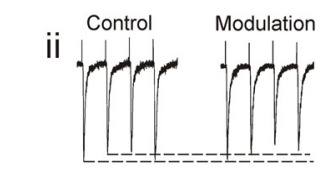

$10 \mathrm{pAL}$

LY 341495 $20 \mathrm{~ms}$

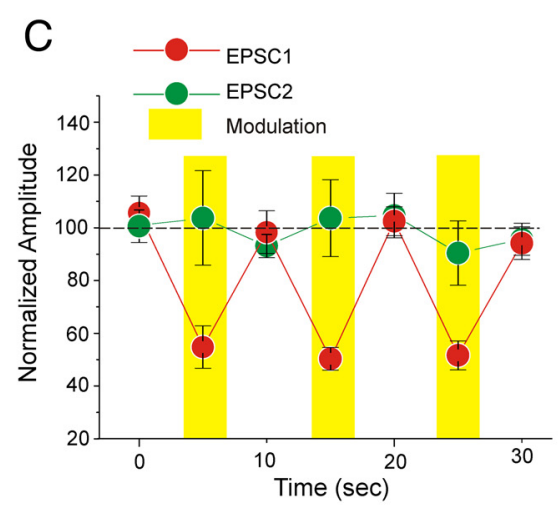

E
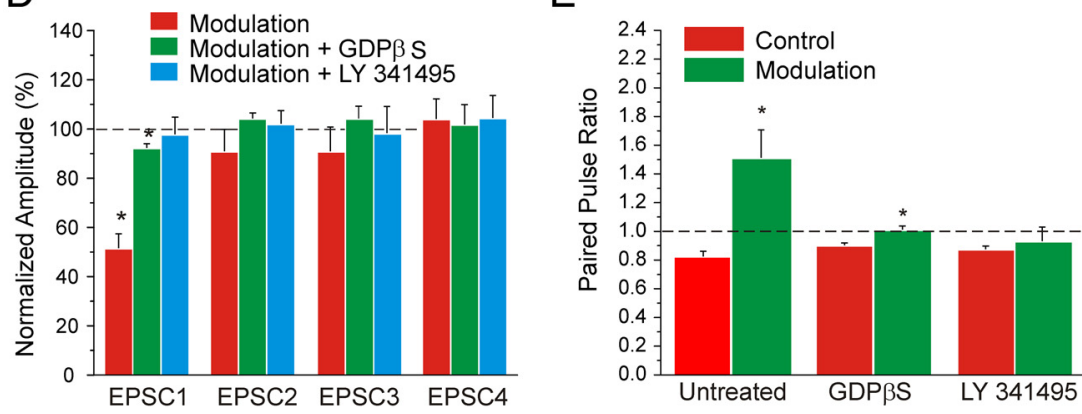

Figure 5. Modulation of input to layers $2 / 3$ via high-frequency stimulation of horizontal connections in layers $2 / 3$ in $V 1$. $\boldsymbol{A}-\boldsymbol{D}$ : Modulation of inputs from layer 4 to layers 2/3. A, Example of traces showing the modulation protocol. Concentric bipolar electrodes are placed in layer 4 (white circle) and layers 2/3 (black circle; $\boldsymbol{A i}$ ) In the Control condition, layer 4 is stimulated with four pulses at $15 \mathrm{~Hz}$ (Aii). In the Modulation condition, layers $2 / 3$ are stimulated first ( 20 pulses, $130 \mathrm{~Hz}$ ), followed by stimulation of layer 4 (4 pulses, $15 \mathrm{~Hz}$ ) $20 \mathrm{~ms}$ after the last stimulation pulse applied in layers 2/3 (Aiii). The effects of the high-frequency stimulation are compared before (black trace) and after (red trace) the application of an antagonist for the Group II metabotropic glutamate receptors. The outward current caused by Group II metabotropic glutamate receptor activation is obscured by the action of ionotropic glutamate receptors activated during high-frequency stimulation. $\boldsymbol{B}$, Same example shown in $\boldsymbol{A}$ focusing on the effects of modulation on the amplitudes of the EPSCs. Stimulation at $15 \mathrm{~Hz}$ causes paired-pulse depression. Modulation decreases the first EPSC and causes a shift of paired-pulse ratio from depression to facilitation (Bi). Bath application of a Group II metabotropic glutamate receptor antagonist prevents the effects of modulation (Bii). C, Graph showing the amplitude of the first two paired EPSCs in five subsequent repetitions of the modulation protocol. Amplitudes are normalized to the average of all the values measured in the control conditions. Modulation decreases the first EPSC ( $p<0.01$ on a RM-ANOVA) but not the second ( $p>0.1$ on a RM-ANOVA). Prior modulation did not affect the subsequent control condition ( $p>0.1$ on a RM-ANOVA). $D$, Graph showing the effects of modulation on the amplitude of the four EPSC. The amplitudes are normalized to the values measured in the control condition. Only the first EPSC is significantly reduced by modulation ( $p<0.01$ on a paired $t$ test). This effect is prevented by subsequent bath application of the Group II metabotropic glutamate receptor antagonist ( $p>0.1$ on a $t$ test). Neurons treated with GDP $\beta$ S are slightly affected by modulation ( $p<0.05$ on a paired $t$ test), but the effect is notably reduced compared to the untreated condition ( $p<0.0001$ on a $t$ test). $\boldsymbol{E}$, Graph showing the effects of modulation on the paired-pulse ratio. Modulation cause a significant increase of the paired-pulse ratio ( $p<0.01$ on a paired $t$ test) that is prevented after bath application of the Group II metabotropic glutamate receptor antagonists ( $p>0.1$ on a paired $t$ test). A minor but significant increase is also present in cells treated with GDP $\beta S$ ( $p<0.01$ on a paired $t$ test).

thalamic and cortical circuits are studied or as other regions in the CNS in other species are explored, many more classes of glutamatergic input may be defined. Whereas the classification of glutamatergic inputs is relatively straightforward, understanding the functional significance of this is not: what different functions are subserved by Class 1 and 2 inputs? Our hypothesis is that Class 1 inputs provide the main information route for thalamic and cor- 
A

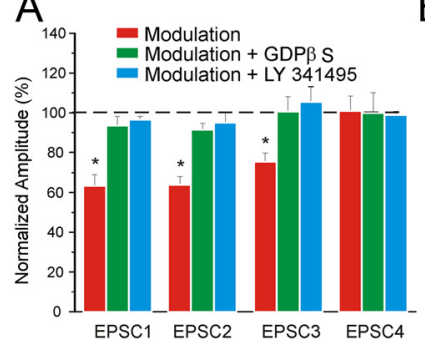

B

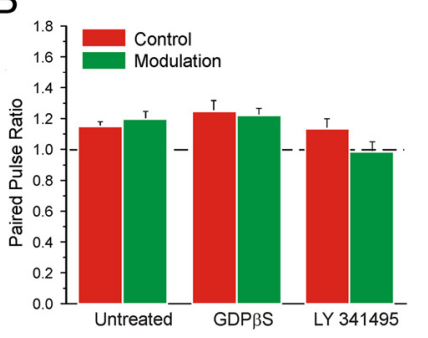

Figure 6. Modulation of input from layers $2 / 3$ to layers $2 / 3$ by stimulation of horizontal connections in layers $2 / 3$ in $V 1 . A$, Graph showing the effects of modulation of inputs from layers $2 / 3$ to layers $2 / 3$ by stimulation of horizontal connections in layers $2 / 3$ in V1. Conventions as in Figure 5D. The first three EPSCs are significantly reduced by modulation $(p<0.01$ on a paired $t$ test for the first two EPSCS, $p<0.05$ for the third EPSC). Modulation has no effect on these amplitudes during either subsequent bath application of a Group II metabotropic glutamate receptor antagonist ( $p>0.1$ on a $t$ test for all EPSCs) or in cells treated with $\operatorname{GDP} \beta S(p>0.1$ on a $t$ test for all EPS(s). All amplitude values are normalized to the value measured in the control condition. $\boldsymbol{B}$, Graph showing the effects of modulation on the paired-pulse ratio. Since both EPSCs are similarly decreased by modulation, the paired-pulse ratio is unchanged ( $p>0.1$ on a $t$ test).

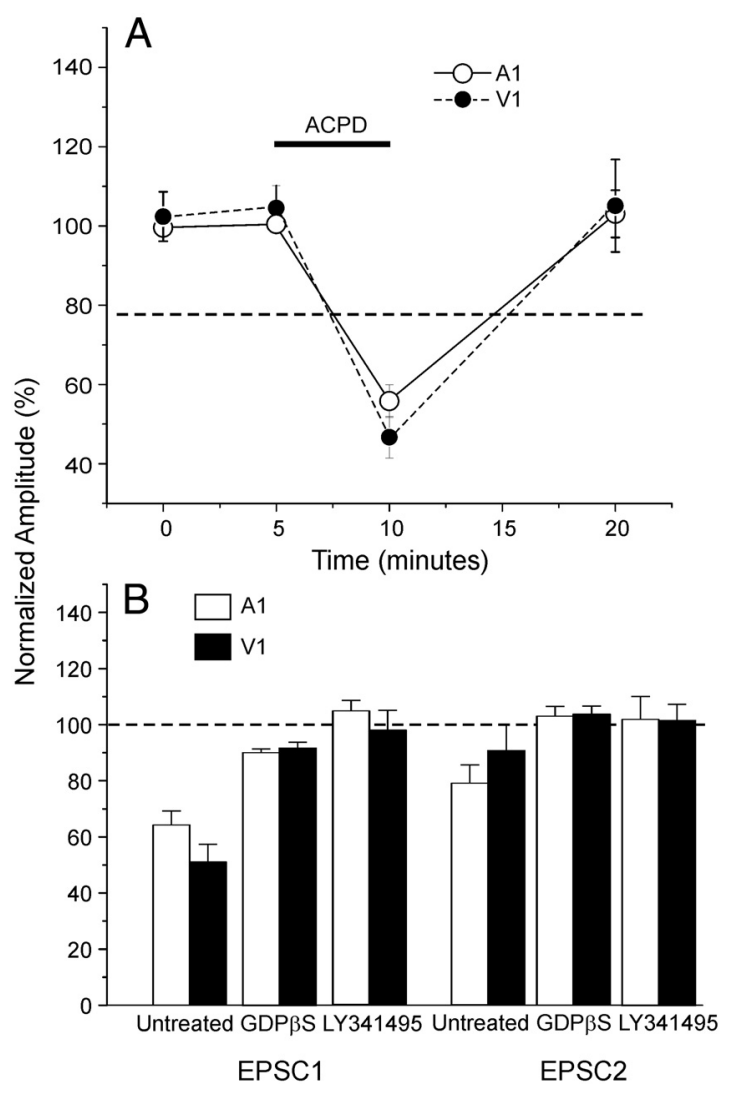

Figure 7. Comparison between A1 and V1. A, Metabotropic glutamate receptor activation also reduces evoked responses in cells of layers $2 / 3$ in $A 1$.Graph shows comparable effects of ACPD on direct photostimulation of cells in layers $2 / 3$ of both $\mathrm{V} 1$ and $\mathrm{A} 1$ while in a low $\mathrm{Ca}^{2+}$ and high $\mathrm{Mg}^{2+}$ bath medium. Amplitudes are normalized to the mean of the base line values. ACPD strongly decreases the EPSCS amplitude in both areas ( $p<0.001$ on a $t$ test). $\boldsymbol{B}_{\text {, Comparison }}$ between $\mathrm{V} 1$ and $\mathrm{A} 1$ of the effects of the modulation protocol for inputs from layer 4 to layers $2 / 3$. The normalized amplitudes of the first two EPSCs are shown. No relevant difference is present comparing the two areas ( $p>0.1$ on a $t$ test).

tical circuits, whereas Class 2 inputs serve a modulatory function. We have explained previously why we believe the properties of these different classes are consistent with these roles (Sherman and Guillery, 2006, 2011), but we emphasize that, especially for cortex, this hypothesis has not been critically tested.

\section{Technical issues}

Much of our data consist of responses evoked by electrical stimulation. We cannot completely rule out the possibility that, in addition to cell bodies and dendrites, axons were stimulated, either antidromically or passing through the stimulation site, leading to additional possible sources of input to connected cells. However, for the following reasons we do not believe that this possibility of inadvertently activating axons significantly affects our conclusions. First, one of our main conclusions, that activation of metabotropic glutamate receptors affects synaptic inputs onto cells in layers $2 / 3$, is supported by activation of inputs via uncaging of glutamate (Fig. 2), an approach that does not activate axons. Second, if we consider our electrical stimulation of layer 4, the main source of axons passing through to layers $2 / 3$ would be thalamocortical axons, because few derive from intracortical sources below layer 4 (Gilbert, 1983; Gilbert and Wiesel, 1983; Dantzker and Callaway, 2000). In our earlier study, we showed that the vast majority of thalamocortical inputs to layers $2 / 3$ are Class 2 (Viaene et al., 2011a), and because our stimulation of layer 4 evoked only Class 1 inputs, it seems unlikely that this included many thalamocortical axons. Third, our electrical stimulation sites in layers $2 / 3$ could have activated axons of passage, evoking a Class 2 input pattern. The major source of these would be thalamocortical axons and projections from other cortical areas, including from the contralateral cortex via the corpus callosum. However, our activation of layers 2/3 evoked mainly Group II metabotropic glutamate receptors, whereas activation of thalamocortical inputs or inputs from another cortical area (V2) activate only Group I metabotropic glutamate receptors (De Pasquale and Sherman, 2011; Viaene et al., 2011a). We thus feel that our conclusions are not significantly affected by the possibility that our electrical stimulation activated axons inappropriately.

\section{Effects of metabotropic glutamate receptors on synaptic transmission}

Our evidence that activation of Group II metabotropic glutamate receptors can affect the amplitude of evoked synaptic potentials in cortex is consistent with other reports showing similar effects with pharmacological manipulation of these receptors (Cahusac, 1994; Taylor and Cahusac, 1994; Reid and Daw, 1997; Beaver et al., 1999; Bandrowski et al., 2001, 2002; Cahusac and Wan, 2007). However, it should be noted that, in studies of the development of such effects, only in younger or immature animals were such effects of activation of metabotropic glutamate receptors on synaptic responses seen, and in adults these effects disappeared (Beaver et al., 1999; Daw et al., 1999; Jin et al., 2001). Many of the reported effects of metabotropic glutamate receptor activation on synaptic processing were carried out in immature animals (Reid and Daw, 1997; Daw et al., 1999; Bandrowski et al., 2001; Jin et al., 2001; Bandrowski et al., 2002). We emphasize that our experiments were carried out in fully adult mice, and so we were not studying a purely developmental process.

\section{General conclusions}

The data reported here support the general view that the classification of glutamatergic inputs into Class 1 and Class 2 can gainfully be applied to cortical circuitry. Here, we identify an input to cells in layers $2 / 3$ from subjacent layer 4 as Class 1 and that from adjacent layers $2 / 3$ as Class 2. Furthermore, in the context of the hypothesis that Class 2 inputs are mostly modulatory, we dem- 
onstrate that activation of Group II metabotropic glutamate receptors via this Class 2 input onto cells in layers $2 / 3$ reduces their EPSC amplitudes activated from layer 4 inputs. This effect is postsynaptic and is more effective at lower rates of layer 4 input. In addition, activation of these Group II metabotropic glutamate receptors produces a hyperpolarization. Together, these two effects of the Class 2 input on its target cells, a hyperpolarization and reduction in EPSC amplitudes evoked from other sources, reduces the flow of information at this stage of cortical processing, although the effect on EPSC amplitude would be relatively small when the layer 4 inputs are firing at higher rates.

There are two consequences of this that are not mutually exclusive. First, the effects that we document of activating metabotropic glutamate receptors are long lasting, and this means that as layer 4 becomes active and activates layers $2 / 3$ for a period of time (hundreds of milliseconds to several seconds), this will modulate as described the layer 4 to layers $2 / 3$ input in adjacent regions, providing a sort of lateral suppression at a columnar scale. Second, this effect might also occur within a column to reset the level of activity flowing from layer 4 to layers $2 / 3$ as a sort of gain control mechanism.

\section{References}

Bandrowski AE, Aramakis VB, Moore SL, Ashe JH (2001) Metabotropic glutamate receptors modify ionotropic glutamate responses in neocortical pyramidal cells and interneurons. Exp Brain Res 136:25-40.

Bandrowski AE, Moore SL, Ashe JH (2002) Activation of metabotropic glutamate receptors by repetitive stimulation in auditory cortex. Synapse 44:146-157.

Beaver CJ, Ji Q, Daw NW (1999) Effect of the group II metabotropic glutamate agonist, $2 R, 4 R$-APDC, varies with age, layer, and visual experience in the visual cortex. J Neurophysiol 82:86-93.

Cahusac PM (1994) Cortical layer-specific effects of the metabotropic glutamate receptor agonist $1 S, 3 R$-ACPD in rat primary somatosensory cortex in vivo. Eur J Neurosci 6:1505-1511.

Cahusac PM, Wan H (2007) Group II metabotropic glutamate receptors reduce excitatory but not inhibitory neurotransmission in rat barrel cortex in vivo. Neuroscience 146:202-212.

Callaway EM (1998) Local circuits in primary visual cortex of the macaque monkey. Annu Rev Neurosci 21:47-74.

Callaway EM, Katz LC (1993) Photostimulation using caged glutamate reveals functional circuitry in living brain slices. Proc Natl Acad Sci U S A 90:7661-7665.

Canepari M, Nelson L, Papageorgiou G, Corrie JE, Ogden D (2001) Photochemical and pharmacological evaluation of 7-nitroindolinyl-and 4-methoxy-7-nitroindolinyl-amino acids as novel, fast caged neurotransmitters. J Neurosci Methods 112:29-42.

Covic EN, Sherman SM (2011) Synaptic properties of connections between the primary and secondary auditory cortices in mice. Cereb Cortex 21:2425-2441.

Dantzker JL, Callaway EM (2000) Laminar sources of synaptic input to cortical inhibitory interneurons and pyramidal neurons. Nat Neurosci 3:701-707.

Daw NW, Reid SN, Beaver CJ (1999) Development and function of metabotropic glutamate receptors in cat visual cortex. J Neurobiol 41:102-107.

De Pasquale R, Sherman SM (2011) Synaptic properties of corticocortical connections between the primary and secondary visual cortical areas in the mouse. J Neurosci 31:16494-16506.

Dudek SM, Friedlander MJ (1996) Intracellular blockade of inhibitory synaptic responses in visual cortical layer IV neurons. J Neurophysiol 75:2167-2173.

Gerber G, Zhong J, Youn D, Randic M (2000) Group II and group III metabotropic glutamate receptor agonists depress synaptic transmission in the rat spinal cord dorsal horn. Neuroscience 100:393-406.

Gilbert CD (1983) Microcircuitry of the visual cortex. Annu Rev Neurosci 6:217-247.

Gilbert CD, Wiesel TN (1983) Clustered intrinsic connections in cat visual cortex. J Neurosci 3:1116-1133.

Jin XT, Beaver CJ, Ji Q, Daw NW (2001) Effect of the group I metabotropic glutamate agonist DHPG on the visual cortex. J Neurophysiol 86:1622-1631.

Lam YW, Sherman SM (2005) Mapping by laser photostimulation of connections between the thalamic reticular and ventral posterior lateral nuclei in the rat. J Neurophysiol 94:2472-2483.

Lee CC, Sherman SM (2008) Synaptic properties of thalamic and intracortical inputs to layer 4 of the first- and higher-order cortical areas in the auditory and somatosensory systems. J Neurophysiol 100:317-326.

Lee CC, Sherman SM (2009) Modulator property of the intrinsic cortical projection from layer 6 to layer 4. Front Syst Neurosci 3:3.

Lee CC, Sherman SM (2010) Topography and physiology of ascending streams in the auditory tectothalamic pathway. Proc Natl Acad Sci U S A 107:372-377.

O’Leary DM, Cassidy EM, O'Connor JJ (1997) Group II and III metabotropic glutamate receptors modulate paired pulse depression in the rat dentate gyrus in vitro. Eur J Pharmacol 340:35-44.

Petrof I, Sherman SM (2009) Synaptic properties of the mammillary and cortical afferents to the anterodorsal thalamic nucleus in the mouse. J Neurosci 29:7815-7819.

Reichova I, Sherman SM (2004) Somatosensory corticothalamic projections: distinguishing drivers from modulators. J Neurophysiol 92:2185-2197.

Reid SN, Daw NW (1997) Activation of metabotropic glutamate receptors has different effects in different layers of cat visual cortex. Vis Neurosci 14:83-88.

Sherman SM, Guillery RW (1998) On the actions that one nerve cell can have on another: distinguishing "drivers" from "modulators." Proc Natl Acad Sci U S A 95:7121-7126.

Sherman SM, Guillery RW (2006) Exploring the thalamus and its role in cortical function, Ed 2. Cambridge, MA: MIT.

Sherman SM, Guillery RW (2011) Distinct functions for direct and transthalamic corticocortical connections. J Neurophysiol 106:1068-1077.

Taylor KE, Cahusac PM (1994) The effects of the metabotropic glutamate receptor agonist $1 S, 3 R$-ACPD on neurones in the rat primary somatosensory cortex in vivo. Neuropharmacology 33:103-108.

Theyel BB, Llano DA, Sherman SM (2010) The corticothalamocortical circuit drives higher-order cortex in the mouse. Nat Neurosci 13:84-88.

Viaene AN, Petrof I, Sherman SM (2011a) Synaptic properties of thalamic input to layers $2 / 3$ in primary somatosensory and auditory cortices. J Neurophysiol 105:279-292.

Viaene AN, Petrof I, Sherman SM (2011b) Synaptic properties of thalamic input to the subgranular layers of primary somatosensory and auditory cortices in the mouse. J Neurosci 31:12738-12747.

Viaene AN, Petrof I, Sherman SM (2011c) Properties of the thalamic projection from the posterior medial nucleus to primary and secondary somatosensory cortices in the mouse. Proc Natl Acad Sci U S A 108:18156-18161. 